\title{
Amyloid imaging for dementia in Canada
}

\author{
Robert Laforce Jr MD PhD, Mohamed Réda Bensaïdane MSc
}

See also page 603 and www.cmaj.ca/lookup/doi/10.1503/cmaj.150508

Competing interests: Both authors report grants from the Société Alzheimer de Québec, Réseau de BioImagerie du Québec and Fonds sur la Maladie d'Alzheimer de l'Université Laval. Robert Laforce also reports nonfinancial support from Navidea and grants from Novartis.

This article has been peer reviewed.

The authors have obtained patient consent.

Correspondence to: Robert Laforce Jr, robert.laforce@ fmed.ulaval.ca

CMAJ 2016. DOI:10.1503 /cmaj.150663
1 lzheimer disease and other types of dementia affect an increasing number of people in our societies and are predicted to be the leading cause of health costs in Canada. Although Alzheimer disease is a clinical diagnosis, trained physicians can diagnose probable Alzheimer disease with a sensitivity and specificity of only $71 \%$ compared with pathology. ${ }^{1}$ Even in cases where detailed neuropsychologic testing, structural brain imaging using magnetic resonance imaging (MRI), and metabolic imaging using positron emission tomography with fluorine18-labelled fluorodeoxyglucose (FDG-PET) is obtained, complex or atypical presentations remain challenging, particularly in younger patients or those with comorbid symptomatology. In such circumstances, diagnostic uncertainty often persists for years, which deprives patients of appropriate treatment and leaves families without closure. Given that amyloid imaging is on the verge of approval from health authorities in Canada, where will this technique fit into current patient care, including primary care?

\section{What is amyloid imaging?}

Amyloid imaging is a technique performed in nuclear medicine. It uses PET ligands that allow in vivo detection of amyloid plaques, a core pathologic feature of Alzheimer disease (positive

\section{KEY POINTS}

- Amyloid imaging allows in vivo detection of amyloid plaques, a core pathologic feature of Alzheimer disease, with high sensitivity and specificity.

- In several clinicopathologic demonstrations, amyloid imaging has been proven to be a reliable method with an important effect on diagnosis and treatment, particularly in complex or atypical presentations in patients younger than 65 years.

- For the moment, amyloid imaging in Canada remains largely a part of clinical trials, but a ligand should soon be available for clinical purposes in Canada.

- Clinicians who wish to obtain amyloid imaging should refer patients to dementia centres with expertise in this technique. amyloid- $\beta[A \beta]$ status). ${ }^{2}$ An example of this is carbon-11-labelled Pittsburgh compound B (PiB), which has a half-life of 20 minutes, but other fluorine-based compounds with a 120-minute half-life also exist. An increasing body of high-quality prospective studies with pathologic confirmation suggest that PiB-PET is sensitive for Alzheimer disease pathology, can distinguish Alzheimer disease from non-Alzheimer disease dementia (e.g., frontotemporal lobar degeneration) and can help determine whether mild cognitive impairment is due to Alzheimer disease. This represents a major advancement in this area, particularly in younger patients $(<65 \mathrm{yr})$ with comorbid conditions.

Moreover, when patients with frontotemporal lobar degeneration mistaken for Alzheimer disease are given acetylcholinesterase inhibitors their condition can worsen, whereas patients with early Alzheimer disease may show relative stabilization in cognition and behaviour for periods ranging from one to three years as a result of this treatment. When amyloid PET was compared with another molecular imaging technique (FDG-PET) in discriminating Alzheimer disease $(n=62)$ from frontotemporal lobar degeneration $(n=45),{ }^{3}$ it was shown to have a higher sensitivity and interrater agreement than FDG-PET in the diagnosis of frontotemporal lobar degeneration $(90 \%, \kappa=0.96, \mathrm{v}$. $78 \%, \kappa=0.72 ; p<0.05)$. In a more recent study, the accuracy of amyloid PET was assessed among 37 pathologically confirmed cases (Alzheimer disease: $n=10$; frontotemporal dementia: $n=24$; prion disease: $n=3){ }^{4}$ The negative predictive value for amyloid PET was $100 \%$, with excellent sensitivity (90\%) and specificity (100\%). ${ }^{4}$ In brief, amyloid imaging has been proven useful, particularly in the differential diagnosis of complex or atypical cases (Figure 1 shows a case illustration).

\section{Who is eligible?}

For the moment, amyloid imaging in Canada remains largely a part of clinical trials. However, similar to what occurred in the United States in 
2012 and the United Kingdom in 2013, health authorities have recently been approached, and a ligand should soon be available for clinical purposes in Canada.

The focus of the Fourth Canadian Consensus Conference on the Diagnosis and Treatment of Dementia, held in 2012, was largely on neuroimaging and other biomarkers, and nine recommendations on amyloid PET were made. ${ }^{6}$ A specialized task force on amyloid imaging in Canada met in the spring of 2015 to update the 2012 recommendations. ${ }^{6}$ The task force discussed indications and, just as important, nonindications of amyloid imaging based on clinical and nonclinical scenarios, with variables including symptoms (typical and atypical), clinical settings, clinical contexts, evidence of cognitive deficits, family history, knowledge of genetic risk of Alzheimer disease and age.

The task force consensus recommendations on the basics of amyloid imaging (e.g., ligands, amyloid binding and the meaning of positivity $\mathrm{v}$. negativity), when to recommend such a test, where to refer a patient, and how to use the test results to orient treatment (i.e., deciding whether to start or discontinue symptomatic medications for symptoms of Alzheimer disease) and plan future care are outlined in Box $1 .{ }^{7}$ Briefly, the task force recommends the use of amyloid imaging in patients with objectively confirmed cogni- tive impairments in whom there is diagnostic uncertainty after a comprehensive clinical evaluation (including mental status testing, laboratory testing and structural brain imaging using MRI), and in whom knowledge of $A \beta$ status is expected to provide a more precise diagnosis and alter management. It is not recommended for use in the initial investigation of cognitive complaints, among other clinical scenarios (Box 1). ${ }^{7}$

\section{What are the possible harms?}

In a recent cross-sectional study that measured amyloidosis and neurodegeneration in 985 participants aged 50-89 years with normal cognition, it was shown that amyloid inevitably accumulates with age. Yet, many people retain normal cognitive function despite substantial amyloid burden. ${ }^{8}$ Hence, the task force does not recommend amyloid imaging in people with normal cognitive function (Box 1). ${ }^{7}$ However, some studies have found negative correlations between $\mathrm{PiB}$ and episodic memory in this group, whereas other studies found no differences across cognitive measures between PiB-positive and PiB-negative controls. Recently, data have shown that participants who have the epsilon 4 allele of the apolipoprotein $\mathrm{E}$ gene $\left(A P O E^{*} E 4\right)$ and have high $A \beta$ showed the highest cognitive decline on the Mini-Mental State Examination. ${ }^{9}$ Altogether,

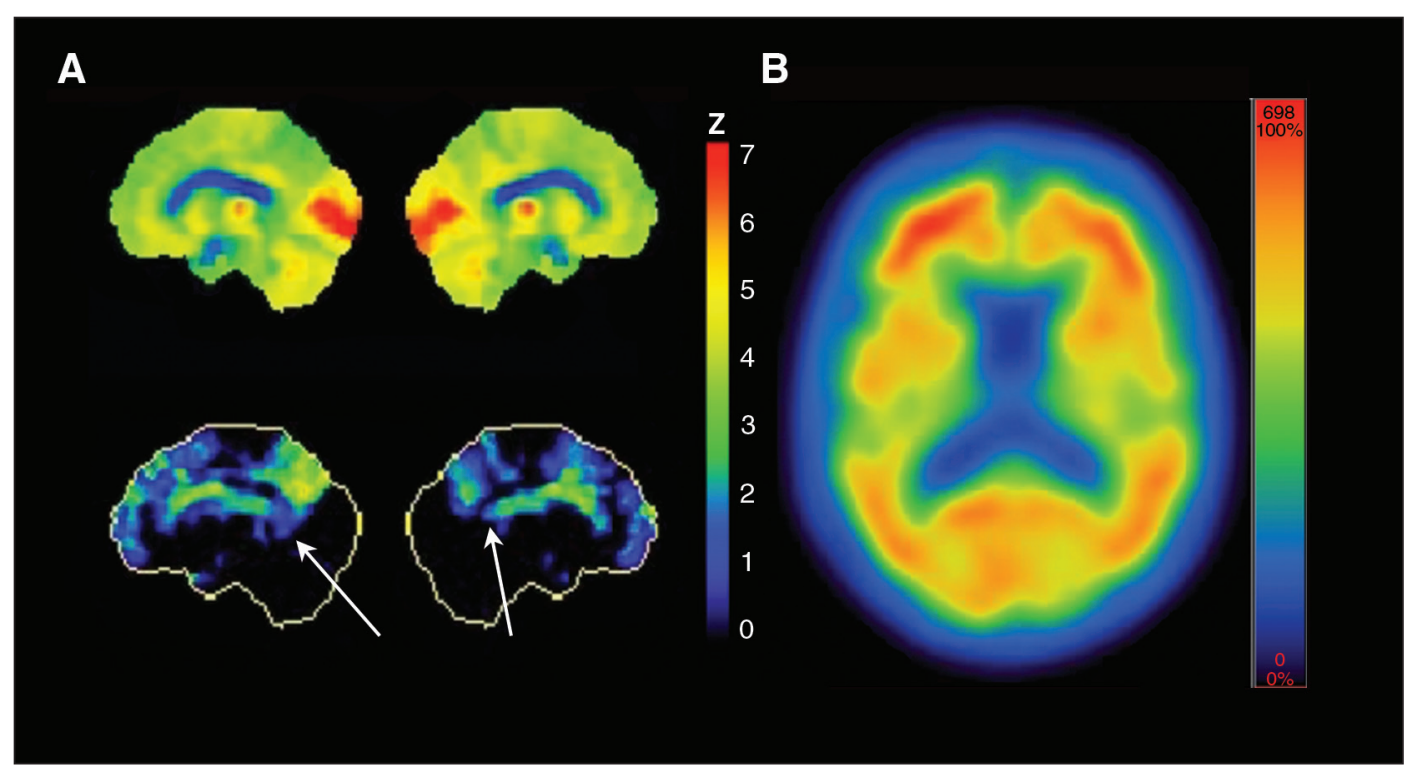

Figure 1: Positron emission tomography with fluorine-18-labelled fluorodeoxyglucose (FDG-PET) (A) and amyloid imaging (B) in a 68-year-old woman with no previous psychiatric history or recent change in medications, who was admitted to hospital because of hallucinations and delusions. She showed severe visuoconstruction deficits and discrete asymmetric motor signs compatible with corticobasal syndrome. This condition can hide various pathologic substrates including corticobasal degeneration (35\%), Alzheimer disease $(23 \%)$, progressive supranuclear palsy (13\%) and frontotemporal lobar degeneration with TDP inclusions (13\%). 2,3 Her FDG-PET scan (A) was incompatible with Alzheimer disease because of sparing of the cingulate gyri (arrows), but amyloid imaging (B) showed substantial diffuse fibrillary deposition of amyloid plaques throughout the brain, a pattern compatible with Alzheimer disease. ${ }^{5}$ She was given an acetylcholinesterase inhibitor and her condition stabilized. 
cross-sectional and early longitudinal data have strengthened the notion that many (although probably not all) PiB-positive controls with normal cognitive function are in a preclinical phase of Alzheimer disease.
Amyloid imaging involves no greater risk than other nuclear medicine examinations. However, disclosure of results can create distress and therefore be harmful. Criteria for appropriate use $^{10}$ suggest the following prerequisites before

\section{Box 1: Recommendations for clinicians on use of amyloid imaging*}

1. Amyloid imaging represents a promising technique in the evaluation of dementia for which much has been learned over the past decade. It is not currently approved for clinical use in Canada. When it becomes available to Canadian clinicians, it must not be considered a routine test:

A. In accord with Appropriate Use Criteria for Amyloid PET, ${ }^{10}$ we recommend its use in patients with objectively confirmed cognitive impairments in whom there is diagnostic uncertaintyt after a comprehensive clinical evaluation (mental status testing, laboratory tests, and structural brain imaging using MRI‡), and in whom knowledge of $A \beta$ status is expected to provide a more precise diagnosis and alter management;

B. Clinicians who wish to obtain amyloid imaging should refer patients to dementia centres with an expertise in this technique, i.e., dementia experts§ with substantial clinical experience and practice in dementia care who work in conjunction with nuclear medicine specialists qualified in amyloid imaging;

C. We strongly recommend against the use of amyloid imaging in cognitively normal individuals or for the initial investigation of cognitive complaints.

2. Physicians should be cautious about interpreting the significance of amyloid test results, i.e. used in isolation this test cannot diagnose $A D, M C l$, or differentiate normal from abnormal aging. When faced with such situations, we recommend they consult with dementia centres with an expertise in this technique.

3. At present, there is no clinical indication for amyloid imaging in:

A. Attempting to differentiate $A D$ from other $A \beta$-associated dementia (e.g., dementia with Lewy bodies, cerebral amyloid angiopathy);

B. Attempting to differentiate between AD clinical variants (e.g., classic amnestic AD vs. posterior cortical atrophy or logopenic variant of primary progressive aphasia);

C. Attempting to differentiate between the various clinical presentations associated with frontotemporal lobar degeneration spectrum of disorders (e.g., behavioral variant frontotemporal dementia vs. progressive supranuclear palsy) to try to define the underlying pathology;

D. Staging the severity of a dementing syndrome.

4. Patients with $\mathrm{MCl}$ represent a highly heterogeneous cohort for which amyloid imaging may be appropriate under specific circumstances. As a general rule, amyloid PET could be considered in $\mathrm{MCl}$ patients for whom the dementia expert has determined that greater certainty about the underlying pathology would alter management (e.g., knowledge of amyloid burden in an individual < 65 years old with confounding circumstances such as depression or other medical disorders, and for whom safety issues at work could have major consequencesף). In such a case, determination of a positive amyloid status could lead to the diagnosis of $\mathrm{MCl}$ resulting from $\mathrm{AD}$, as opposed to a nondegenerative condition, and have significant repercussions for future care and planning.

5. The actual process of undergoing an amyloid scan and the implications associated with disclosure of the results should be taken very seriously because this can be highly stressful for patients and families. To maximize safety and effectiveness of disclosing results, we recommend adopting parts of the sequence recently developed in cognitively normal older adults participating in $A D$ prevention studies. This format includes an educational session with clinical scenarios before the scan, assessment of mood and willingness to receive the results, and a formal face-to-face disclosure session in which results are discussed along with their diagnostic and prognostic implications.

Note: $A \beta=$ amyloid $\beta, A D=$ Alzheimer disease, $\mathrm{MCl}=$ mild cognitive impairment, $\mathrm{MRI}=$ magnetic resonance imaging, $\mathrm{PET}=$ positron emission tomography.

*Reprinted, with permission, from Laforce et al. ${ }^{7}$ OCambridge University Press.

tSubjects with diagnostic uncertainty after a comprehensive tertiary memory clinic evaluation are also named "complex/atypical cases" and often include AD variants, non-AD dementias (e.g., frontotemporal lobar degeneration), nonprogressing AD, or patients with comorbid and nondegenerative conditions (e.g., depression, substance abuse, atypical bipolar disorder).

\#In accord with the Canadian Consensus Conference on the Diagnosis and Treatment of Dementia $4,{ }^{6}$ structural brain imaging using MRI means a "Head MRI - Dementia Protocol," which includes: (1) coronal $T_{2} /$ fluid-attenuated inversion recovery and (2) axial susceptibility-weighted imaging, diffusion-weighted imaging, as well as three-dimensional $T_{1}$ sequences. Structural brain imaging using MRI should always be performed before amyloid imaging as a positive scan in typical AD distribution in a patient with dementia can be secondary to cerebral amyloid angiopathy.

$\S$ Dementia expert: a physician with substantial clinical experience and practice in dementia care. Expertise in dementia is acquired through formal training and clinical experience in neurology, psychiatry and geriatric medicine; however, not all dementia experts have expertise in amyloid imaging and/or work in conjunction with nuclear medicine specialists qualified in amyloid imaging, hence the recommendation to refer to a dementia centre with expertise in this technique.

ПSafety issues and the notion of major consequences: this is determined on a case-by-case basis with all significant clinical information. An example of this could be an emergency medical technician, paramedic or a lead squad firefighter, etc. 
conducting such a test: an objectively confirmed cognitive impairment; Alzheimer disease as a possible diagnosis but uncertain even after a comprehensive assessment; and knowledge of presence or absence of Alzheimer disease pathology is expected to increase diagnostic certainty and alter management. An algorithm illustrates several clinical situations in which amyloid imaging may be helpful ${ }^{11}$ (Appendix 1, available at www.cmaj.ca/lookup/suppl/doi:10.1503/ cmaj.150663/-/DC1). The sequence of investigation before amyloid imaging in the algorithm is congruent with the recommendations of the Fourth Canadian Consensus Conference on the Diagnosis and Treatment of Dementia. ${ }^{6}$ Further validation of the algorithm is underway.

\section{What is the evidence so far?}

A growing body of literature supports the clinical utility of amyloid imaging for the differential diagnosis in patients with an atypical presentation and an uncertain diagnosis. One study followed 229 patients who underwent amyloid imaging as part of their diagnostic workup. ${ }^{12}$ The authors found a $54.5 \%$ diagnostic change and a $21.6 \%$ mean increase in clinicians' diagnostic confidence. The impact on the diagnostic process was consistently greater in difficult cases where initial diagnostic confidence was low, which has major implications for a cohort of patients who are often younger than 65 and still active in the workforce. Indeed, several dementia experts have argued that an accurate diagnosis helps direct therapy (i.e., avoid unnecessary or undesired cholinesterase inhibitors or memantine prescriptions), determine a better care plan (which considers patient safety and minimizes the risk of preventable complications), and enables patients to participate in legal and financial planning. ${ }^{2}$

More specifically, literature suggests that amyloid imaging has an effect on management in three specific domains: medication management, the ordering of unnecessary tests and the value of knowing what is wrong..$^{10}$ In as many as $86.9 \%$ of patients, it increases use of cholinesterase inhibitors by $17.7 \%$ in the amyloid-positive group, it decreases such prescriptions by $23.3 \%$ in the amyloid-negative group (where an inhibitor can exacerbate dementia symptomatology), and it decreases planned additional investigations (e.g., structural or metabolic imaging, neuropsychologic testing and lumbar puncture). ${ }^{12}$ More important, it clarifies the diagnosis in young patients with atypical presentations who can now plan for the future according to their health condition.

Amyloid imaging is not the sole biomarker that may signal underlying Alzheimer disease pathology. A decade or more before the appear- ance of dementia symptoms, and possibly before amyloid accumulation is detectable by PET, changes in cerebrospinal fluid (CSF) can appear and correlate with brain atrophy in older people with normal cognitive function. Researchers have compared amyloid imaging with CSF biomarkers of Alzheimer disease in the same study and found that CSF A $\beta 1-42$ analyzed consecutively in routine clinical practice at an accredited laboratory can be used with high accuracy to determine whether a patient has normal or increased cortical $\mathrm{A} \beta$ deposition and so can be valuable for the early diagnosis of Alzheimer disease. ${ }^{7}$ Other groups replicated these findings using crosssectional and longitudinal designs. ${ }^{7}$ The utility of CSF in diagnosing unclear dementia syndromes, such as those on the frontotemporal lobar degeneration spectrum, is less clear. At the moment, CSF variability across techniques and centres is such that it limits proper, confident interpretation of the results. Due to the absence of appropriate laboratory infrastructure in Canada, or consensus as to where the samples should be sent for analysis, $A \beta 1-42$, total tau and phosphorylated tau 181 have no clinical utility in Canada (i.e., are not recommended for clinical practice), although they are part of research protocols in observational and therapeutic studies. International efforts to standardize CSF biomarkers for Alzheimer disease, notably with more reliable enzyme-linked immunosorbent assay (ELISA) techniques, are currently underway.

\section{What can we expect in the future?}

Much is expected from the recently launched Imaging Dementia - Evidence for Amyloid Scanning (IDEAS) Study, a \$100-million open-label longitudinal study involving about 18500 US Medicare beneficiaries. In this venture, patients with diagnostically uncertain cases of mild cognitive impairment and atypical dementia will be referred by physicians of all specialties, including those in primary care, to be scanned to determine whether knowledge of amyloid status leads to substantial changes in patient management and whether this translates into improved medical outcomes.

In addition to enhancing the diagnostic process of atypical dementia syndromes and allowing earlier treatment, we can expect amyloid imaging to be a major contributor to future advancements and discoveries in the field of Alzheimer disease and the natural evolution of dementias. For example, amyloid imaging has served as a secondary outcome measure in clinical trials of Alzheimer disease with disease-modifying agents such as the antiamyloid monoclonal antibodies bapineuzumab and solanezumab. Recently, a study has shown 
brain amyloid reduction and slowing of cognitive decline after one year of treatment with aducanumab, a human immunoglobulin $\mathrm{G}$ subclass 1 monoclonal antibody against a conformational epitope found on $A \beta \cdot{ }^{13}$ Hence, confirming the amyloid-positive status of patients in clinical trials (e.g., the Anti-Amyloid Treatment in Asymptomatic Alzheimer's [A4] Study) will be of the utmost importance for validation of therapies.

Finally, clinicians who wish to obtain amyloid imaging are expected to refer patients to dementia centres with an expertise in this technique (Box 1). However, because of the very nature of their front-line work, family physicians will likely be expected to answer questions from patients and their families about this testing, as well as follow up on the anticipated impact of amyloid imaging on patient care (i.e., changes in medication management and future planning). This holds an even greater importance given that ligand approval from Canadian health authorities is imminent.

\section{References}

1. Beach TG, Monsell SE, Phillips LE, et al. Accuracy of the clinical diagnosis of Alzheimer disease at National Institute on Aging Alzheimer's Disease Centers, 2005-2010. J Neuropathol Exp Neurol 2012;71:266-73.

2. Laforce R, Rabinovici GD. Amyloid imaging in the differential diagnosis of dementia: review and potential clinical applications. Alzheimers Res Ther 2011;3:31.

3. Rabinovici GD, Rosen HJ, Alkalay A, et al. Amyloid vs FDGPET in the differential diagnosis of AD and FTLD. Neurology 2011;77:2034-42

4. Rabinovici G, Lehmann M, Rosen H, et al. Diagnostic accuracy of amyloid and FDG PET in pathologically-confirmed dementia. Neurology 2014;82:S8.005.
5. Bensaïdane MR, Fortin M, Damasse G, et al. Clinical utility of amyloid imaging in a complex case of corticobasal syndrome presenting with psychiatric symptoms. J Neurol Disord 2014; 2:1-4.

6. Gauthier S, Patterson C, Chertkow H, et al. Recommendations of the 4th Canadian Consensus Conference on the Diagnosis and Treatment of Dementia (CCCDTD4). Can Geriatr J 2012; 15:120-6.

7. Laforce R, Rosa-Neto P, Soucy JP, et al. Canadian consensus guidelines on use of amyloid imaging in Canada: update and future directions from the Specialized Task Force on Amyloid Imaging in Canada. Can J Neurol Sci 2016 Feb 26:1-10. [Epub ahead of print]

8. Jack CR, Wiste HJ, Weigand SD, et al. Age-specific population frequencies of cerebral beta-amyloidosis and neurodegeneration among people with normal cognitive function aged 50-89 years: a cross-sectional study. Lancet Neurol 2014;13:997-1005.

9. Mormino EC, Betensky RA, Hedden T, et al. Amyloid and APOE epsilon4 interact to influence short-term decline in preclinical Alzheimer disease. Neurology 2014;82:1760-7.

10. Johnson KA, Minoshima S, Bohnen N, et al. Appropriate use criteria for amyloid PET: a report of the Amyloid Imaging Task Force, the Society of Nuclear Medicine and Molecular Imaging, and the Alzheimer's Association. Alzheimers Dement 2013;9:e-1-16.

11. Vandenberghe R, Adamczuk K, Dupont P, et al. Amyloid PET in clinical practice: its place in the multidimensional space of Alzheimer's disease. Neuroimage Clin 2013;2:497-511.

12. Grundman M, Pontecorvo MJ, Salloway SP, et al. Potential impact of amyloid imaging on diagnosis and intended management in patients with progressive cognitive decline. Alzheimer Dis Assoc Disord 2013;27:4-15.

13. Keller D. Finally, a big win for a monoclonal in Alzheimer's. Proceedings from AD/PD 2015: International Conference on Alzheimer's and Parkinson's Diseases; 2015 Mar. 18-22; Nice (FR). Medscape; 2015. Available: www.medscape.com/view collection/33320 (accessed 2015 July 31).

Affiliations: Clinique Interdisciplinaire de Mémoire (CIME), Centre Hospitalier Universitaire de Québec; Faculty of Medicine, Université Laval, Québec, Que.

Contributors: Both authors contributed substantially to the conception and design of the article, and drafted and revised the article. Both authors gave final approval of the version to be published and agreed to act as guarantors of the work. 